Seraient publiés ceux des quatre manuscrits suivants, en voie d'achèvement, qui seront prêts les premiers:

(a) Volumes vi et vir de Melbourne, préparés par l'observatoire de Sydney;

(b) zones $+34^{\circ}$ et $+35^{\circ}$, préparées par l'observatoire de Paris.

Compte tenu d'un reliquat de fonds disponibles, après les publications en cours, une attribution de 9000 dollars est demandée.

\title{
Commission 24
}

I. La Commission 24 demande en subvention une somme de rooo dollars, au plus, pour la publication d'un catalogue de toutes les étoiles de l'Hémisphère Austral qui ont un mouvement propre connu supérieur à 0.2 par an (le catalogue comporte environ 9000 étoiles).

2. La Commission 24 recommande instamment que les observatoires possédant de grands réflecteurs (au moins I mètre) envisagent un programme sérieux de déterminations de parallaxes trigonométriques pour des étoiles très faibles.

\section{Commission 25}

I. La Commission demande au Comité exécutif de former une sous-commission de la Qualité des Images.

2. La Commission demande au Comité exécutif de réunir les deux sous-commissions $25 a$ et $25 b$ sous le titre 'Sous-commission des Magnitudes Etalon' (les membres des deux sous-commissions étant réunis).

3. La Commission présente les recommandations suivantes:

I. Pour normaliser la photométrie en deux couleurs, les observateurs devront employer

(a) une modification du système international photographique-photovisuel, ou toien $(b)$ le système $B, V$ de Johnson et Morgan, ou bien $(c)$ dans l'hémisphère austral, le système I953 $\mathrm{S}$.

2. Pour normaliser la photométrie en trois couleurs, les observateurs devront utiliser, pour le présent, le système U, B, V, de Johnson et Morgan.

3. Les observateurs sont instamment priés d'utiliser un filtre (tel que Schott GGI3) pour éliminer les radiations de longueur d'onde inférieure à $3800 \AA$, chaque fois qu'ils déterminent des magnitudes 'bleues' ou 'photographiques'.

\section{Commission 26}

I. La Commission décide que le futur Catalogue Général d'étoiles doubles visuelles se fera sur la base du projet van den Bos (Trans. de l'U.A.I. 8, 386) et demande aux Bureaux centraux $\mathrm{N}$. et $\mathrm{S}$. de disposer en conséquence leur matériel.

Les principes du projet pourront recevoir des amendements de détail et devront être complétés sur certains points non tranchés (ex.: limites du catalogue); le tout en accord avec les opinions déjà exprimées soit à Rome, soit dans les réponses au referendum de H. M. JEFFERS et après une nouvelle consultation générale.

2. La Commission approuve la création de la Circulaire d'Information sous sa forme actuelle et demande à P. Muller d'en assurer, jusqu'à nouvel ordre, la composition et la diffusion au mieux des besoins.

\section{Commission 27}

De nombreux travaux ont été faits récemment sur les variables d'amas globulaires. De telles études qui mettent en lumière les propriétés caractéristiques des variables d'amas en relation avec les propriétés de l'amas auquel elles appartiennent, ont une grande influence sur la cosmologie et l'évolution des amas globulaires: à ce titre, elles méritent un intérêt spécial. Pour ces motifs, la Commission 27 demande la formation d'une souscommission des étoiles variables d'amas globulaires. On propose qu'elle soit présidée par le Dr Helen Sawyer Hogg, qui a fait sur ce sujet des travaux étendus. 